\title{
Identification and Characterization of Information-Networks in Long-Tail Data Collections
}

\author{
Mostafa M. Elag ${ }^{\mathrm{a}}$, Praveen Kumar ${ }^{\mathrm{a}, *}$, Luigi Marini ${ }^{\mathrm{b}}$, James D. Myers ${ }^{\mathrm{c}}$, \\ Margaret Hedstrom ${ }^{c}$, Beth A. Plale ${ }^{\mathrm{d}}$ \\ ${ }^{a}$ Ven Te Chow Hydrosystems Laboratory, Department of Civil and Environmental \\ Engineering, University of Illinois, Urbana, IL, USA \\ ${ }^{b}$ National Center for Supercomputing Applications, University of Illinois at \\ Urbana-Champaign, Urbana, IL, USA \\ ${ }^{c}$ School of Information, University of Michigan, Ann Arbor, MI, USA \\ ${ }^{d}$ School of Informatics and Computing Director, Data to Insight Center, Indiana \\ University Bloomington, Bloomington, IN, USA
}

\section{Abstract}

Scientists' ability to synthesize and reuse long-tail scientific data lags far behind their ability to collect and produce these data. Many Earth Science Cyberinfrastructures enable sharing and publishing their data over the web using metadata standards. While profiling data attributes advances the Linked Data approach, it has become clear that building informationnetworks among distributed data silos is essential to increase their integration and reusability. In this research, we developed a Long-Tail InformationNetwork (LTIN) model, which uses a metadata-driven approach to build semantic information-networks among datasets published over the web and aggregate them around environmental events. The model identifies and characterizes the spatial and temporal contextual association links and dependencies among datasets. This paper presents the design and application of the LTIN model, and an evaluation of its performance. The model capabilities were demonstrated by inferring the information-network of a stream

\footnotetext{
${ }^{*}$ Corresponding author. kumar1@illinois.edu

Preprint submitted to Environmental Modelling 83 Software March 24, 2017
}

(C) 2016. This manuscript version is made available under the Elsevier user license 
discharge located at the downstream end of the Illinois River.

Keywords: Long-tail data; Information-Networks; Linked-Data;

Cyberinfrastructure; Environmental Data, Data-intensive science.

\section{Introduction}

Scientific data collected or produced by individuals and small groups are generally targeted to address specific scientific issues and often have limited geographic or temporal coverage $[17,44,14]$. These data are usually irreplaceable, expensive to reproduce, infrequently reused, and have been defined as long-tail data $[42,19,27]$. As the volume of long-tail data increases, the scientific community is developing and maintaining CyberInfrastructures (CIs) to manage and share their data, including the Consortium for the Advancement of Hydrologic Science, Inc., Hydrologic Information System (CUASHI-HIS) [43], DataOne [32, 3], Sustainable Environmental Actionable Data (SEAD) [33], and the Critical Zone Observatory Integrated Data Management System (CZOData) [47]. These CIs rely on the Service-Orientated Architecture (SOA) to advance the Data as a Service (DaaS) principle, which allows publishing and delivering data over the web via standard coding of their metadata using markup languages and web services [21]. In DaaS, a data node refers to any self-contained dataset that has a unique identifier and information profile, which explicitly depicts its attributes, behavior, and relationship with other nodes [27]. A large number of data nodes together constitute a data collection, i.e., a virtual aggregation of data nodes coming from different CIs. Identification of the contextual association links and dependencies among long-tail data nodes has immense value for increasing their 
integration and reusability, which are required to address multidisciplinary science challenges and advance scientific exploration $[23,18]$.

Contextual Association Links (CALs) are primitive and essential types of links that refer to potential associations among data nodes due to proximity in space, time, problem context, or any other user-defined criteria. CALs are required to build geoscience information-networks among data nodes distributed across different CIs and link data nodes with related environmental events. An Information-Network (IN) describes the CALs across the many attributes of independent long-tail data nodes. For example, understanding changes in the spatiotemporal patterns of sediment and contaminant fluxes in a river due to climate and land use change requires an information-network that links hydrologic, water quality, biological, atmospheric, sediment, and nutrient monitoring data [28]. Similarly, understanding the changes in cropping pattern, which refers to changes in an area cultivated with various crops over a period of time, requires identification of the CALs among current and historical hydrologic, atmospheric, and geochemical data from soil samples along with sociocultural and economic data. Currently, building informationnetworks among distributed data nodes for such multidisciplinary studies is hindered by heterogeneity in coordinate systems, scales, provenance, units, variables, providers, users, file formats, and scientific contexts [19, 17, 38].

Integration of distributed data nodes is a tedious and time-consuming process because a scientist has to search across distributed CIs, analyze heterogeneous information profiles associated with data nodes, identify the types of relationships among data nodes, and annotate the data nodes with their relationships. A long-tail data collection may include data that are generated 


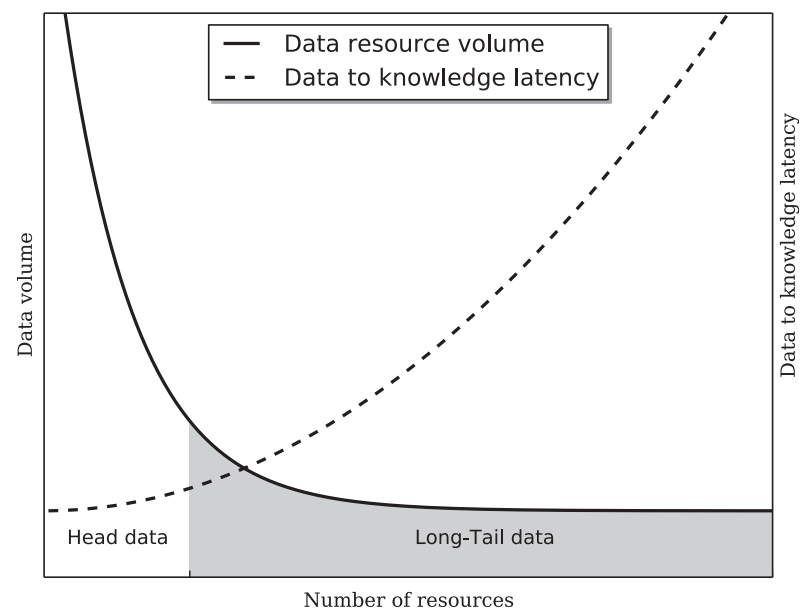

Figure 1: The Grey zone refers to the scientific long-tail data that make up the bulk of the scientific research output. It has an exponential production rate compared to "Big data", which are produced and maintained by very large projects and agencies. The knowledge required to reuse long-tail data is lagging far behind the data production (adapted from [17] and [19].)

from a variety of sources such as scientific experiments, field observations, model simulations, or derived from other data nodes. Diversity in data types, formats, and data models are the primary challenges in seamless integration of distributed data $[21,27,22]$. In addition, advances in instrumentation, sensing, computational approaches, and information exchange technologies, are rapidly increasing the production rate of such data. The combination of heterogeneity and high production rates of data increases the data-toknowledge latency, that is, the production of knowledge by harnessing data lags far behind the data production itself [17, 15, 12]. This latency hinders the integration and reusability of data (Figure 1). Thus, an efficient data integration strategy requires multiple syntactic and semantic translations to manage across different domain CIs with various data models and identify CALs among distributed data nodes. 
Indeed, overcoming the data-to-knowledge latency is essential for large multidisciplinary science projects such as the Critical Zone (CZ) science, where scientists are required to mange diverse data nodes from different CIs. Because many of the existing CIs have their data structure and data models implemented, one solution to overcome the challenge of data-to-knowledge latency is to build information-networks. The inclusion of new data nodes in a long-tail collection and/or modification of old data nodes continuously changes the IN, thereby, creating emergent characteristics with the potential to identify novel dependencies among the data nodes. Analysis of the emergent IN enables us to immediately identify the new data node in a broader context of other data nodes, while at the same time enhancing the value of existing data nodes. We argue, along with others [8, 24, 46], that building an IN of long-tail data will minimize the data-to-knowledge latency, potentially alleviate the problem of underutilized data resources, and foster the emergence of useful knowledge. This research focuses on two specific contextual association links — space and time - which identify and characterize the spatial and temporal relationships among scientific long-tail data using their information profiles. However, the model presented here is applicable for building CALs among data based on a broader set of their attributes such as information about data providers.

Prediction of a CAL between two data nodes is key to characterizing their emergent dependencies and establish an IN. Typical approaches for link prediction follow a content-based paradigm that relies on statistical inference of data connectivity based on the analysis of their numerical values. This paradigm ranges from feature-based grouping, which trains a binary classifier 
using a set of predefined features to classify data, to probabilistic graphical models, which use a Bayesian model to compute the joint-probability of a connection between data nodes [2]. Many real networks including social and biological networks adopt this paradigm [30, 45]. Applying the content-based approaches to predict long-tail data linkages requires a considerable amount of effort and time to manually reformat large volumes of heterogeneous data nodes, where the source of heterogeneity cannot be anticipated in advance. In addition, sophisticated statistical techniques are required to minimize uncontrolled errors resulting from the interference of many factors in identifying the statistical dependency among data nodes such as sample size, missing data, and type of inter-variable dependencies (linear or nonlinear) [34]. Therefore, there is a pressing need for a new context-based approach to solve these issues through rapid understanding of the spatial and temporal CALs among long-tail data.

In this research, we combined Information Retrieval (IR) methods, which are provided by scientific CIs with the Context-Aware Recommender System 130 (CARS) to build the Long-Tail Information-Network (LTIN) model. First, information retrieval methods retrieve information profiles relevant to a specific search context. Then, CARS tailors the recommendation based on analyzing the retrieved information profiles using rules [1]. The present work is primarily focused on the challenge of building a recommendation model (engine), which is aware of the information profiles of distributed data nodes and can identify their CALs based on spatial and temporal rules. The model is introduced with two flavors: Long-Tail Information-Network (LTIN) full and lite. LTIN-full registers the information retrieval method of each CI and 
builds an IN among data nodes, which are available in the data collection. build an IN between a single data node (given by the user) and nodes in the data collection on the fly. The model provides a semantic information graph that annotates its data nodes with their CALs, and characterizes the strength of the predicted CALs based on a strength matrix. The annotations are compliant with the Semantic Web standard format to allow consistent interaction among the data nodes distributed over the Internet [16].

The remaining sections of the paper are organized as follows. Section 2 provides a design overview of the Information-Network. Section 3 describes the components of the Long-Tail Information-Network model and its implementation. Section 4 gives an example application. Finally, Section 5 summarizes this work and discusses possible directions for future research.

\section{Information-Network Design Overview}

Information-Network (IN) is a graph-based network that connects two data nodes using an edge (relationship). Each node is defined using a Unique Resource Identifier (URI), and has an information profile, which describes its spatial, temporal, and variable attributes. An IN is an undirected graph (bidirectional), where the presence of an edge between two linked nodes in one direction implies the presence of a reverse edge. An undirected graph is used to ensure the development of bi-directional edges between nodes. Edges in the IN encode a variety of information, such as the association between two data nodes occurring in the same spatio-temporal window, whether the nodes have a known relationship such as spatial topological relationships or an unknown 
relationship that needs to be investigated. Each edge is described with a label to define the type of the CAL between nodes and weight to identify its strength.

To identify and characterize the CALs among data nodes, we assume nodes in a data collection can be potentially linked, and the task of the LTIN model is to predict the existence, type, and weight of edges. We have considered two types of logic patterns for representing the relationships among data nodes: (i) association: patterns that link data nodes that have spatial and temporal topological relationships, and (ii) sorting: patterns that organize data nodes according to their temporal features. For example, to understand how the water quality of a river will change in response to climate and land use change over time and space, the LTIN model builds and stores the information graph associated with the river data node, which represents its location. The LTIN model associates the river data node with the data nodes that represent the stream gauges that lie in the river, the water quality monitoring sensors that are located within the same watershed, and the terrain, soil, meteorology, and climate data nodes that are spatially related to the river. Within the river's information-network, the LTIN characterizes the chronological relationships between data nodes, such as the temporal dependency of nitrate concentration in the river on nitrogen application in the related soils.

The LTIN model analyzes three fundamental and embedded information concepts of any Earth Science data: (i) space that defines the spatial area, which is covered by a data node, including the location and the shape of coverage that varies from a point to multi-polygons; (ii) time, which describes 
the time window of the feature that is represented by the data node; and (iii) variable, which identifies the symbols, scientific names, and units of the each data node [31]. While the LTIN model can ingest and analyze extra concepts such as the spatial concepts proposed by [26], the current version of the LTIN model is limited to the three fundamental concepts because most of the data models are using these concepts. The LTIN model enables the identification of spatial concepts introduced by [26], including neighborhood, which relates different phenomena through location; network, which specifies the spatial connectivity among data nodes; and event, which aggregates data around environmental events. Given a set of $n$ data nodes (Vertices) $V=\left\{v_{i}\right\}_{i=1}^{n}$, and $m$ logic predicates $P=\left\{p_{i}\right\}_{i=1}^{m}$, the objective of the LTIN model is to infer the relationships between two data nodes based on their attributes $[(u$, $v) \in V]$, and establish a network graph $G(V, E)$ where $E$ is the vector of $k$ edges $E=\left\{e_{i}\right\}_{i=1}^{k}$ that are inferred from applying the logic predicate:

$$
\begin{array}{r}
P\left[p_{i}, \ldots p_{m}\right] \rightarrow V\left[v_{i}, \ldots v_{n}\right] \Longrightarrow G(V, E):= \\
{\left[u, v \in V(G) \mid \exists e \in E\left[e_{i}, \ldots e_{k}\right]: e=<u, v>\right]}
\end{array}
$$

Predicate $P$ maps vertices $V$ to graph $G(V, E)$ such that for $u$ and $v$ belonging to $V(G)$, there exists an edge $e \in E$ where $e$ links $u$ and $v$. Each logic predicate defined in $P$ is applied to test the binary relationships between vertices grouped in $V$. An edge $e$ is identified between two data nodes $u$ and $v$ to generate a triple $u, e$, and $v$. Then, this inferred triple is added to the IN graph $G$.

The LTIN model uses a metadata-driven approach to process a high num- 
ber of data nodes programmatically over the web. The metadata-driven approach analyzes the information profiles associated with data nodes. It minimizes the human inference in processing of information over the web $[29,35]$. In addition, processing of information profiles over the web is easier and more efficient than processing data files because information profiles are often accessible, do not require access control, and their sizes are small, as measured in bytes [41]. Many applications in environmental science are built using metadata-driven approaches [e.g., 29, 9, 10, 39]. Developing an IN includes four challenges: (i) What is the core structure of the information query template? (ii) What are the logic rules (arguments) required to identify a CAL between two information profiles? (iii) How do we allow the dynamic updating of the Information Graph $G$ (e.g., insertion and/or deletion of new edges in the Graph)? And (iv) How do we annotate the data with the predicted CALs? The next section elaborates on techniques used to address these challenges.

\section{Implementation of the Long-Tail Information-Network Model}

Figure (2) describes the architecture of the Long Tail-Information-Network (LTIN) model, which consists of three sequential layers. The first layer retrieves information profiles (fields and values) from CIs' endpoints such as Application Programming Interfaces (APIs), which are registered and indexed in the model. Then, it normalizes the fields and values of the incoming information profiles to be compatible with the requirements of the subroutines in the link prediction layer. The normalized information profiles are passed to the second layer, Link Prediction, which infers new edges between 
data nodes by analyzing the values of the incoming information profiles using logic predicates stored in $P$. Finally, the third layer, Annotation and Visualization, provides an annotation for each edge and weight to produce an interactive graph that allows the navigation between nodes.

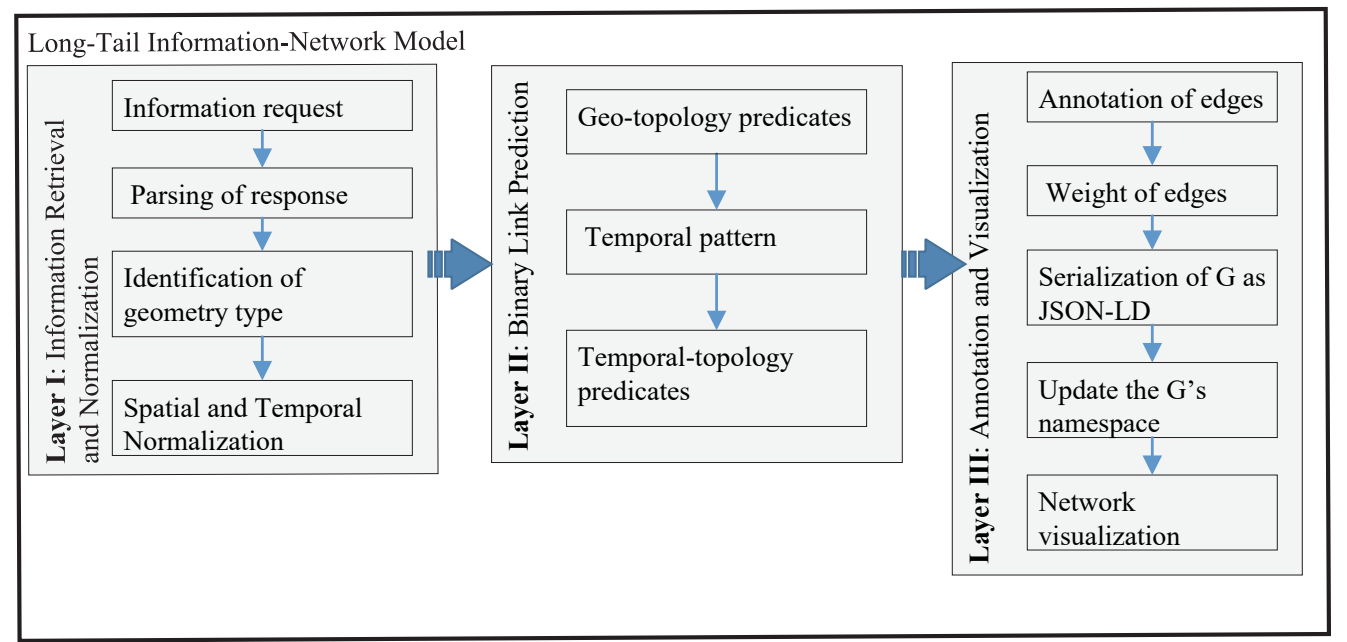

Figure 2: The three layers of the LTIN model and their functionality.

The LTIN produces an online information graph, where the number of nodes and edges are expected to expand rapidly. Given that graph construction is computationally expensive, we decided to precompute the graph and store it in an online graph database. We implemented the Jena-Triple store DataBase (Jena-TDB) as a graph database for the IN because it provides a high-performance triple store and SPARQL endpoint to manipulate the information-network. Currently, the IN is updated on a daily basis and is available at (http://hcgs.ncsa.illinois.edu/gsis/read?graph= information_network). 


\subsection{Information Retrieval and Normalization}

The LTIN model requests from endpoints associated with each registered CI the space, time, and variable attributes of its data nodes. First, the space attribute is defined by: coordinates and Spatial Reference system IDentifier (SRID), which is a unique value used for identifying data geographic projection. Second, the time attribute of data, which is identified by its temporal horizon including Start Datetime, End Datetime, and Time Zone. Finally, the variable attribute is defined by the variable tags associated with a data node.

Since information profiles vary among CIs in their structure, metadata standards, access method, and response format, we mapped the fields available at each endpoint to those required by the LTIN model and indexed the endpoints of each CI. These maps overcome the syntactic heterogeneity among CIs to automatically parse their information profiles. The indexed list of CIs needs to be manually updated when a new CI is added to the list or when the structure or response format of a registered CI is changed. The current endpoints list of the LTIN model includes meta-information, which is information about the endpoints, provided by three types of CIs, including DataOne, Clowder, Geostreams, CUASHI-HIS, and GeoServer. DataOne and Clowder represent CIs that curate file-based data nodes, Geostreams and CUASHI-HIS store time-series observation data nodes, and GeoServer curates spatial data. First, DataOne (https://www.dataone.org/) comprises a distributed network of data centers, where each center is represented as a member node. DataOne provides a RESTFul API, which serializes the metadata of datasets that can be retrieved from each member node. DataOne's 
API uses different metadata formats to encode the information profiles of its data nodes. Second, Clowder (https://clowder.ncsa.illinois.edu) is a scalable data management system for sharing, organizing, and analyzing long-tail data. It serves as a backbone for many long-tail data management systems such as Sustainable Environmental Actionable Data (SEAD, http://sead-data.net/). Clowder provides a RESTFul API, which serializes the information profiles of data nodes using JavaScript Object Notation (JSON). Third, Geostreams is a RESTFul interface that provides access to time and geospatial referenced data nodes and codes their information using JSON format. Geostreams is deployed by several systems including the SeaGrant Great Lakes Monitoring project (SGGLM, http:// greatlakesmonitoring.org/), which curates environmental data collected through the Great Lakes resources [40]; the Great Lakes to Gulf project (GLTG, http://www.ngrrec.org), which is a virtual observatory that monitors water resources from the Mississippi River and featured watersheds in the Mississippi River Basin; and the Intensively Managed LandscapesCritical Zone Observatory project (IML-CZO, http://data.imlczo.org), which curates hydrological, ecological, geological, and biological data in three sites in Illinois, Iowa, and Minnesota. Fourth, GeoServer curates the Hydrologic Unit Codes (HUCs) that identifies a hydrologic feature and its order (for example, stream order), for the IML-CZO, GLTG, and SGGLM respectively and provides their information through a RESTFul API. Finally, CUAHSIHIS (https://www . cuahsi.org) hosts hydrologic time-series data, especially for US, and provides the WaterOneFlow ("WOF") web services for searching and retrieving the stored data, where the services' responses are wrapped 
using WaterML, which is an eXtensiable Markup Language (XML) format for encoding hydrologic observation data [6,37].

Normalization of the retrieved information profiles ensures the consistency of the received information with the LTIN algorithm's input requirements. It compares the spatial and temporal reference of the received information with the LTIN model's references, and converts the anomalous nodes to match the model's references. LTIN includes two types of normalization: spatial and temporal. The first ensures that all data nodes are projected to the same geographic reference. In this study, the European Petroleum Survey Group:4326 (EPSG:4326) reference was selected as a reference SRID for the model [20]. Temporal normalization checks the consistency of the structure and format of the received time attributes. The time structure is defined by time interval, which has a start and end time. The time format is described by the International Standard ISO 8601, which specifies the numeric representation of data and time that can be used directly by computer interfaces. The temporal normalization function matches the time interval format of a data node with the reference time format. Finally, the graph vertices $V(G)$ and their normalized attributes are sent to the link prediction layer.

\subsection{Binary Link Prediction}

Information profiles of data nodes are turned into Contextual Association Links (CALs) in the binary link predication layer, which includes two sets of logic rules to identify and characterize the relationships among data nodes. These sets are: (i) the geo-topological set, which characterizes the spatial dependency between data nodes based on their geographic position and geometrical shape type, and (ii) the temporal-variable set, which characterizes 
the temporal dependency between data nodes based on their time horizon and associated variable tags.

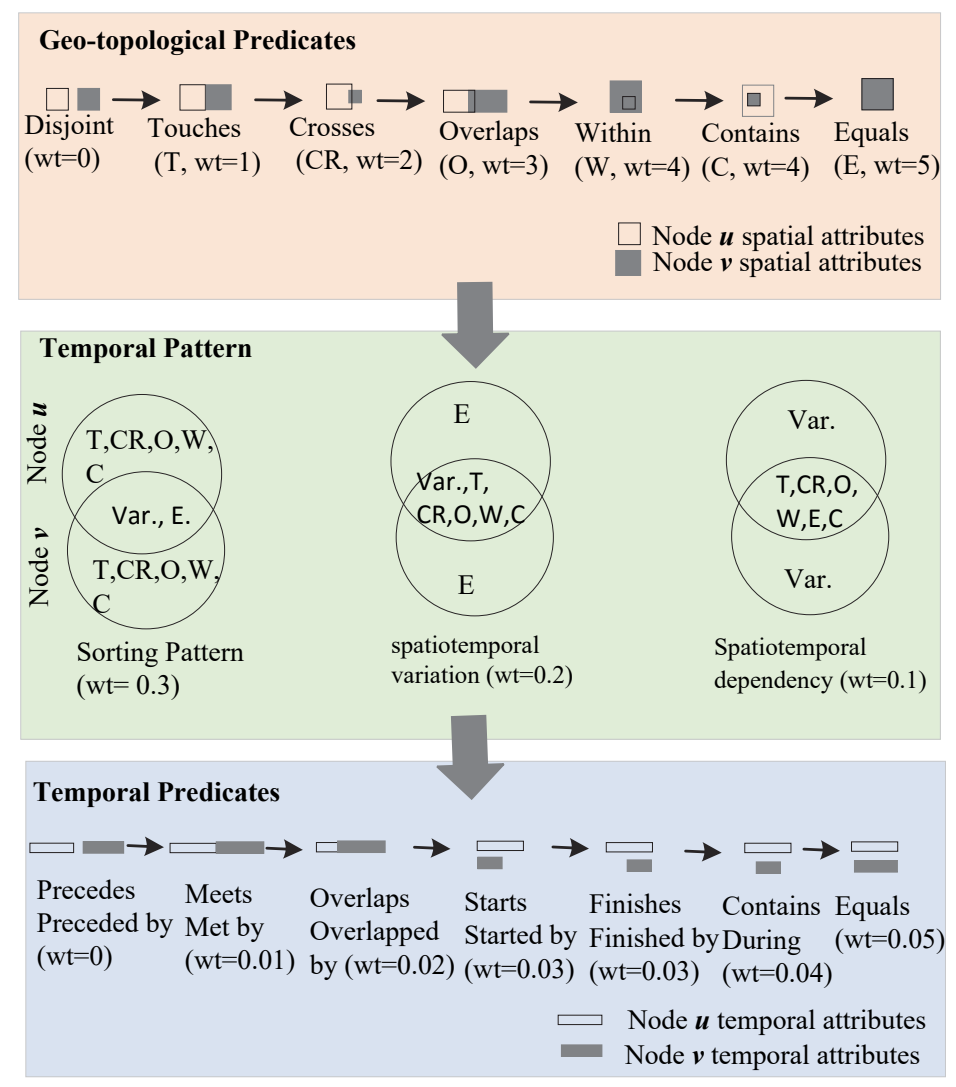

Figure 3: Overview of the binary link prediction layer.

\subsubsection{Geo-topological Predicates}

Six geo-topological predicates defined by the Open Geospatial Consortium (OGC) are implemented in the LTIN model to identify the geo-topological relationships among data nodes (Figure 3). These predicates are abstracted by [11]: disjoint, touches, crosses, within, overlaps, and equals. LTIN's geotopological subroutine either uses the geometric shape of a data node, if the 
Table 1: Matrix of OGC geo-topological predicates used to identify the spatial relationships among the geometric types of data nodes.

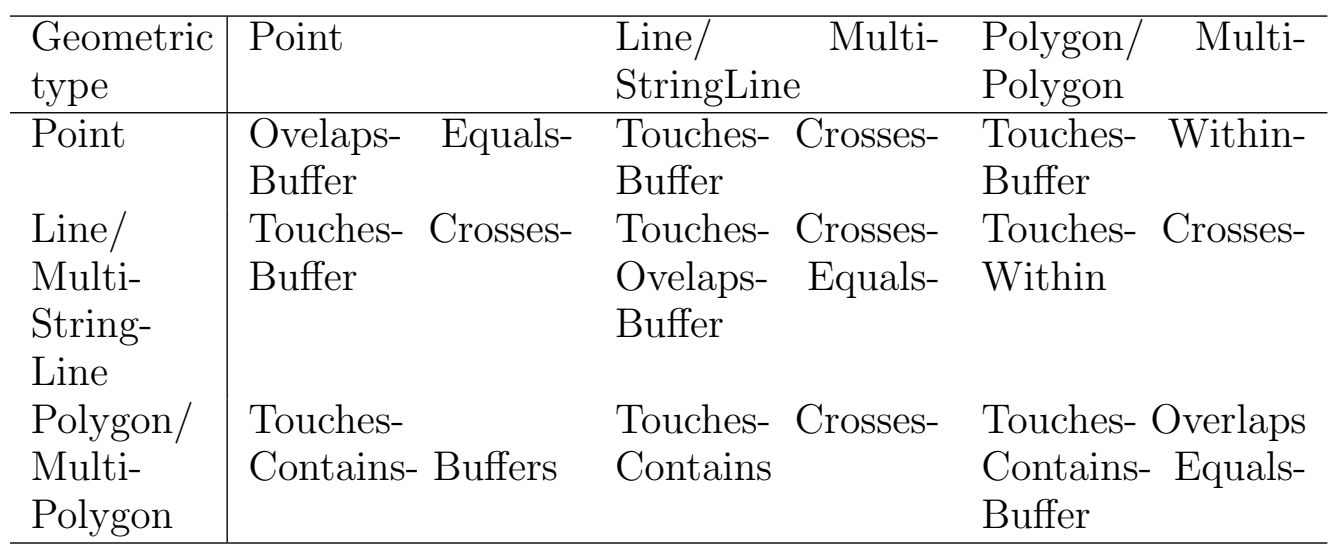

shape is defined in the node's information profile, or identifies the geometric shape of a node using its coordinates and assigns a geometric type to the node's identifier. The geometric shapes that can be processed by the LTIN model include point, line, polygon, multiline string, and multipolygon. Table 1 defines the matrix of OCG geo-topological predicates that are used to identify the spatial contextual relationships among data nodes. A qualifier, which specifies the geometric type of a data node (e.g., gml: Point), is added to the source and target data nodes to precisely characterize their geometric type. Since these geo-topological predicates may occur among different geometric shapes (e.g., equal may occur between two points or two polygons), we used geometric shape qualifiers to precisely describe the geo-topological predicate.

The weight of a geo-topological edge between two nodes is determined based on the edge type and according to the values defined in Figure 3, 
where Equal and Touches are the strongest and weakest edges respectively. To minimize the number of developed edges, the LTIN model filters out the Disjoint relationships. We created the eStrength function (Equation 2) to eliminate the Disjoint relationships and produce the "weighted" graph $G=(V, E, w)$, where the function is applied to $G=(V, E)$ to assign a value from each inferred edge to a value based on the weight values (vals) set that is illustrated in Figure 3.

$$
\text { eStrength } \rightarrow G(V, E) \Longrightarrow G(V, E, w):=w \in \text { Vals }
$$
represent different variables (Figure 3). The spatiotemporal dependency pattern links data nodes that are temporally and spatially correlated regardless of their variable tags. It recommends a temporal edge but, cannot assert the presence of this edge. 
We developed a temporal reasoning algorithm to identify the temporal dependency pattern and the temporal topological relationship among data nodes. The algorithm filters the data nodes based on their geo-topological edges and variable tags, and then assigns nodes for the suitable temporal by the geo:sfEquals predicate and have the same variable tag are assigned to the chronological sorting pattern. We implemented the logic rules, which were defined by Allen [4] to characterize the temporal topological relationships among data nodes. Figure (3) illustrates the 13 predicates that are used to characterize the temporal topological relationship between the two data nodes $u$ and $v$ and the weight associated with each predicate. There is an inverse relationship for each predicate except the equal predicate, which has no inverse. The predicates are defined by a function named temporal predicates and applied for the three patterns in Algorithm (1). Besides the characterization of the temporal relationship between data nodes, LTIN prevents the development of transitive relationships between nodes, i.e., if the time horizon of Node $A<$ Node $B<$ Node $C$, then LTIN will not create a temporal topological relationship between Node $A$ and Node $C$. This functionality is applied only for temporal sorting of data nodes that share the same location and variable tag, to prevent the creation of unnecessary relationships between data nodes. The relationship between Node $A$ and Node $C$ can be identified by tracing the data graph associated with each data node.

An edge strength is represented using a two decimal place number, where the integer describes the geo-topological relationship, the first decimal defines the temporal pattern, and the second identifies the temporal topological 
$\overline{\text { Algorithm } 1 \text { Temporal reasoning over data nodes that have geo-topological }}$ edges.

Given: $G(V, E)$ where $V_{T}=\left(t_{\text {start }}, t_{\text {end }}\right), V_{\text {Var }}=\left(v_{1}, \ldots, v_{n}, . ., v_{m}\right), e \in$ E[Touches, Crosses, Overlaps, Within, Equals $]: e=\langle u, v\rangle$

Required: identify the temporal relationships between two data nodes.

if $e=$ Equals \& $u_{V a r} \cap v_{V a r}=\left\{\mathrm{x} \mid \mathrm{x} \in V_{V a r}\right\}$ then \# chronological sorting Temporal Pattern= Sorting;

Edge Strength $+=0.3$;

Temporal Predicate $=$ Allen's temporal reasoning $(u, v)$;

Edge Strength $+=[0: 0.05]$

end if

if $e \neq$ Equals \& $u_{\text {Var }} \cap v_{\text {Var }} \neq \emptyset$ then \#spatiotemporal variation

Temporal Pattern=Spatiotemporal variation;

Edge Strength $+=0.2$;

Temporal Predicate $=$ Allen's temporal reasoning $(u, v)$;

Edge Strength $+=[0: 0.05]$

end if

if $e \in E \& u_{V a r} \cap v_{V a r}=\emptyset$ then \#spatiotemporal dependency

Temporal Pattern=Spatiotemporal dependency;

Edge Strength $+=0.1$;

Temporal Predicate $=$ Allen's temporal reasoning $(u, v)$;

Edge Strength $+=[0: 0.05]$

end if 
relationship between two data nodes. Figure 3 depicts the values that are assigned for each predicate. For example, 5.28 is the weight of an edge between two data nodes that has a "geo:Contains" relationship and are characterized by a spatiotemporal dependency temporal pattern and have "time:Meets" relationships, i.e., data nodes represent different variable tags, and start time of the source node meets the end time of the target node.

\subsection{Annotation and Visualization}

The objectives of this layer are the annotation of data nodes and production of an interactive IN graph. This layer receives the "weighted" graph $G=$ $(V, E, W)$ then serializes the graph using JavaScript Object Notation-Linked Data (JSON-LD) to develop a semantic IN. The output of the LTIN model is a labeled and weighted graph, where the Unique Resource Identifier (URI) of a data node is used as its identifier, and the URI of a predicate is used to annotate the relationship between nodes. LTIN uses the standards recommended by the World Wide Web Consortium (W3C) for representing the IN, including the Web Annotation Ontology (AO, https://www.w3.org/ns/oa) to describe the annotation of a data node, the OGC Simple Feature Access standards (OGC-SFA, http://www.opengeospatial.org/standards/ sfa) to define the geo-topological relationships between data nodes, and the Time-Ontology Web Language (Time-OWL, https://www.w3.org/TR/ 2016/WD-owl-time-20160712/) to describe the temporal topological relationships between data nodes.

To allow the visualization of the IN over the web, we developed a Javascrpit that can be implemented in any Hyper Text Markup Language (HTML) page to invoke the LTIN-Lite, fetch the data graph associated with a given 
data node, and render in an interactive diagram that allows a user to navigate among data nodes using their URIs. The JavaScript is named as InformationNetwork (IN.js) and relies on the Data-Driven Documents (D3.js) library to render the node's graph.

420

\subsection{Validation of LTIN Prototype}

An instructive unit test is presented in this section to validate the accuracy of the LTIN model and quantify how it scales as the number of data nodes increases. The test uses data from SeaGrant Great Lakes Monitoring (SGGLM, http://greatlakesmonitoring.org). This project includes data from networks of sensors that collect field observations, including water quality, surface hydrology, and air quality in the Great Lakes. SGGLM encodes each observation record as shown in List 1, where the observation has an ID and information profile. Observations of the SGGLM are collected from 119 sites over the Great Lakes, including Superior, Huron, Michigan, Ontario, and Erie Lakes, 96,821 observation records were available from 1990 at the time of this test. SGGLM considers each observation as an individual data node and publishes each observation with a unique URI.

List 1: Example of infromation profile associated with a stream observation in SGGLM.

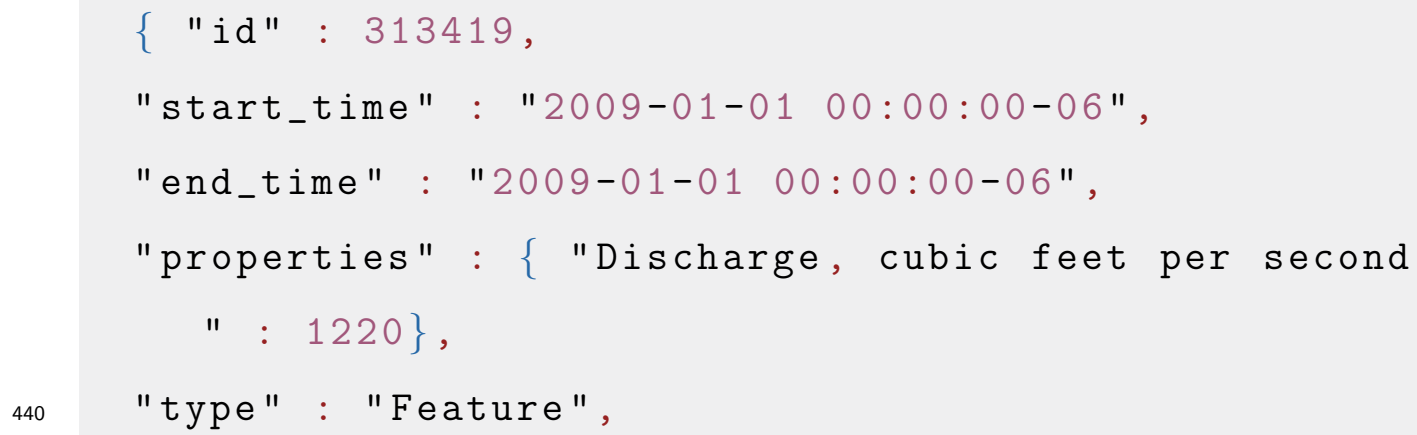


"geometry" : \{ "coordinates" : [ -92.4188018,46.7 $032765,0]\}\}$

To validate the model accuracy, we compared the edges predicted by the LTIN model among the SGGLM's observations with the already known relationships between a sensor ID and its observations, as well as their chronological order. For simplicity, we will demonstrate the IN associated with the Lake Superior data node, which is a shape file representing its boundaries and is stored in the GoeServer of SGGLM. The LTIN model identifies and characterizes two types of spatial relationships among the SGGLM's observations. First, 36927 data nodes share 21 distinct spatial locations within Lake Superior, where the number of these locations are equivalent to the number of sensors. In this test, two types of geo-topological relationships are developed among data nodes: a geo:sfEqual edge is developed between data nodes that share the same geographic position, and a geo:Within relationship between the Lake Superior data node and all the other data nodes that are located within Lake Superior's geographic boundaries. The temporal pattern between data nodes are characterized by the chronological sorting pattern because nodes are aggregated in separated geographic locations (sensor locations). Figure $4 \mathrm{~b}$ represents an excerpt of an IN that has developed among nodes sharing the location of sensor $(\mathrm{SI}=762)$, where nodes are temporally sorted and each node has a geo:equals relationship with the rest of the other nodes. This confirms that the LTIN model was able to predict the spatial context of received data similar to their actual configuration.

One important consideration that must be evaluated for the LTIN model is its performance as the number of the incoming data nodes in an IN in- 


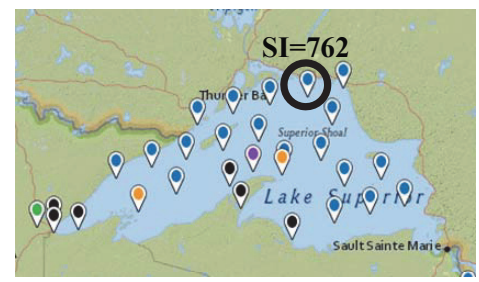

(a) The geographic locations of data nodes that are available in Lake Superior from SGGLM. The number associated with each data node represents its record ID.

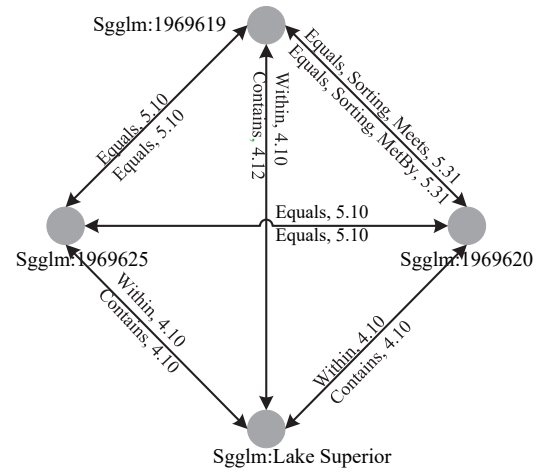

(b) IN of data nodes that share the sensor ID: 762 .

Figure 4: An excerpt of an Information-Network that LTIN inferred in Lake Superior.

creases. The goal of this experiment is to quantify in a general sense the execution time, which is the time consumed by a CPU to execute each layer of the LTIN model. The model was tested five times. In each test, a new data load from a registered CI was added to the existing load (Figure 5). We used a single machine with i7-350M CPU and 8.00 GB RAM and 215 Mbps Internet download speed.

Figure 5 illustrates the execution time for each layer of the LTIN model under different data load. The "Information Retrieval and Normalization" and "Binary Link Prediction" layers follow a power trend, which clearly demonstrates the advantage of providing a precomputed graph to avoid long execution time over the web. Given the high-speed Internet that was used in the scalability test, we can infer that both layers consume most of the model execution time in overcoming syntactic and semantic heterogeneity among the information profiles coming from different CIs. In contrast, the "Annotation and Visualization" layer follows a linear trend with a constant slope as 


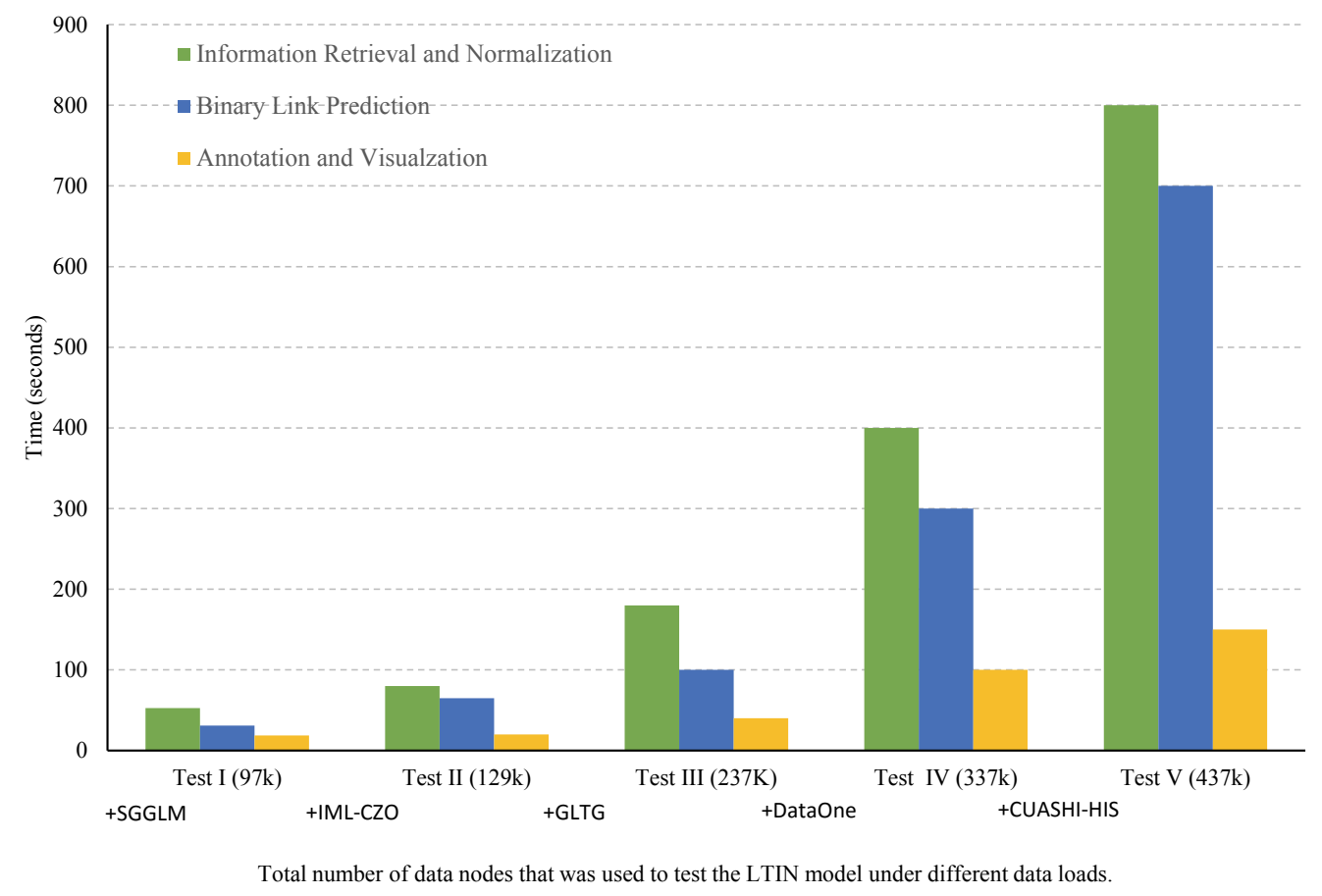

Figure 5: The execution time for each layer of the LTIN model for various data loads.

the number of data loads increases. When interpreting the results we must also consider that most of the data are time series data type, meaning the LTIN model inferred a considerable number of triples $(\cong 2,500,000$ triples $)$ to represent the temporal dependency among data nodes. In the case of LTIN-lite, this analysis suggests that as the number of data nodes increases beyond 300,000 nodes, a new machine needs to be deployed to minimize the execution time of the LTIN model. The current deployment of the LTIN-lite created for this study is a very light-weight deployment that aims to demonstrate the capabilities of the engine. Future research will focus on optimizing the performance of the link prediction layer. 
Table 2: Input type and format for the LTIN-lite version.

\begin{tabular}{|c|c|c|}
\hline Attribute & Filed & Format \\
\hline Space & $\begin{array}{l}X_{\text {min }} \\
Y_{\text {min }} \\
X_{\text {max }} \\
Y_{\text {max }} \\
\text { SRID }\end{array}$ & List $<$ String $>$ \\
\hline Time & $\begin{array}{l}T_{\text {start }} \\
T_{\text {end }} \\
T_{\text {zone }}\end{array}$ & List $<$ String $>$ \\
\hline Variable & Variable Tag & List $<$ String $>$ \\
\hline CIs & default or name & List $<$ String $>$ \\
\hline
\end{tabular}

\subsection{LTIN-lite}

LTIN-lite identifies the IN for a particular data node. It is an interactive web service that is built on the LTIN model and requires a user to provide the space, time, and variable information for the source data node. The service is available at (http://hcgs.ncsa.illinois.edu/gsis/in/space= $<$ Space $>$ \&time $=<$ Time $>\&$ var $=\langle$ Variable $\rangle \& \mathrm{CI}=\langle\mathrm{CI}\rangle)$. The type and format of the inputs for LTIN-lite are specified in Table 2. To simplify the use of the service, we designed it to filter the search CIs according to a user's preference (Table 2). Many application can be built on top of LTIN-lite including the IN.js, which is a web application that encodes the semantic relationships among data nodes in HTML and allows a user to visualize the IN over the web.

Figure 6a illustrates how the annotation coming from the LTIN-lite is encoded in an HTML page, which is used for demonstration purposes. The web page represents a stream discharge time series (source data node), which 
is measured at the downstream of Lake Erie Western Basin. In this graph, the stream discharge node (SGGLM:128) is linked to nodes that represent estimated global precipitations (DataOne: kubi.lifemapper.sdm.layers.513), climate parameters (DataOne: ORNL 173.3), and gridded concentration of soil microbial biomass (DataOne: farshid25.41.1) including Carbon (C), Nitrogen $(\mathrm{N})$, and Phosphorus $(\mathrm{P})$. The aggregation of climate parameters and concentration of soil microbial biomass around stream discharge events, assists in exploring the influence of climate and discharge on the biodegradability of organic matters in soil.

Figure 6b illustrates the response of LTIN-lite, which semantically enriches the information profile of (SGGLM:128) with its IN coming from DataOne CI. In this example, the stream discharge ID is semantically identified (e.g., time: Interval) and linked to distributed data nodes. Embedding the stream's IN in its HTML is known as the Microformats approach, which is the quickest and simplest way to semantically enable long-tail data over the web $[25,12,5]$. The semantic links among nodes allow (i) human users to navigate, for example, to the web page of climate parameters (ORNL 173.3) data node, which is available in DataOne CI; and (ii) machines to programmatically understand the CALs between the two data nodes. The current implementation of IN.js represents a data node with its source CI and URI ("source CI: URI"), and allows the nodes to be grouped by color, annotation, and edge weight. It is straightforward to change the attribute that represents a data node in another HTML because, all the information associated with the data node is stored in its IN. 


\section{Information-Networks}

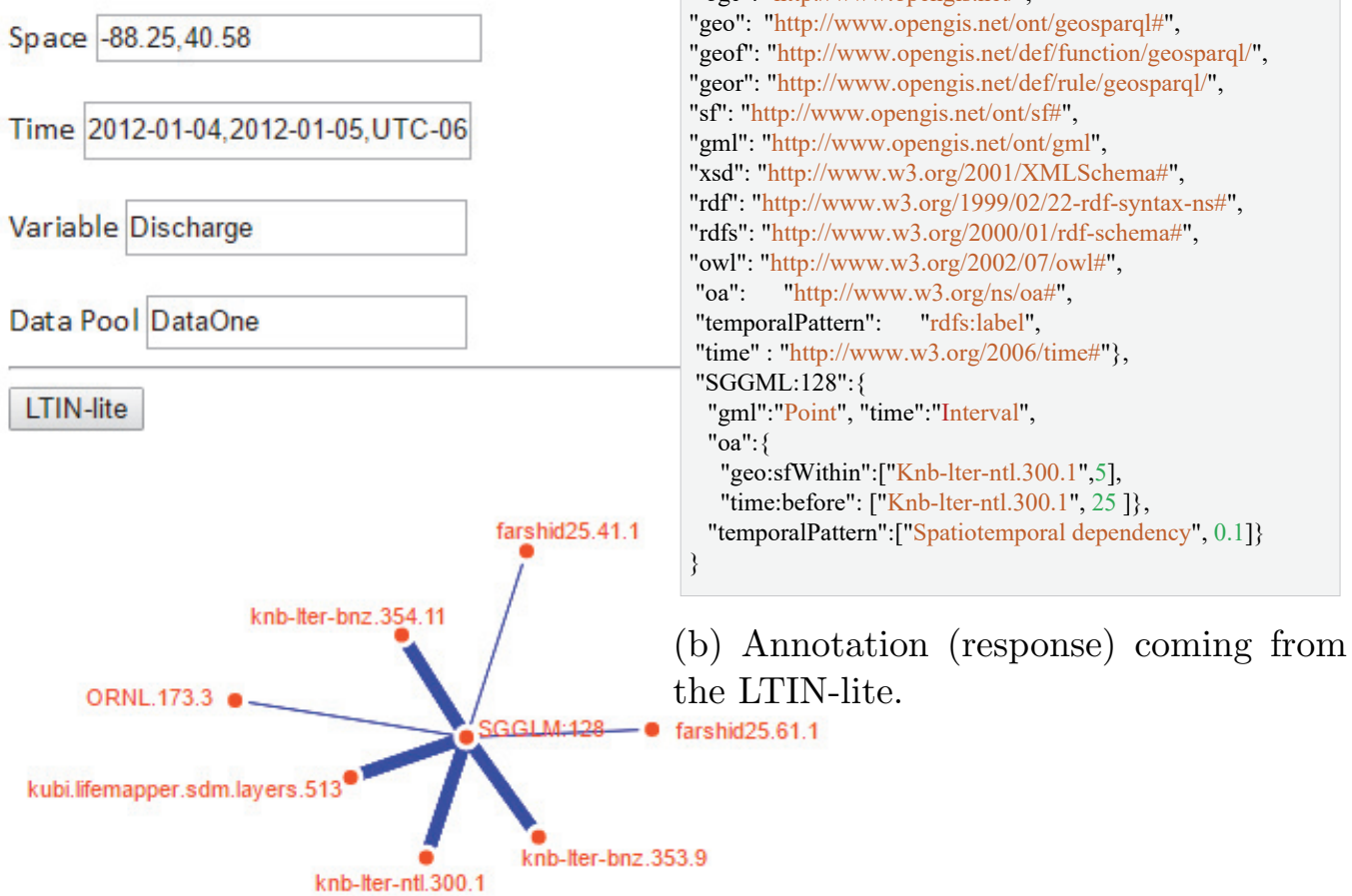

(a) Implementation of IN.js in the Data

node's HTML and visualization of its IN.

Figure 6: An excerpt of the IN inferred by LTIN-Lite for "SGGLM:128" as a source data node and DataOne as a targeted CI. The IN is rendered in a test HTML using IN.js, which invokes the LTIN-Lite and implement the annotation (response) in the HTML . The edge width represents the strength of th relationship between data nodes. Bold edges identify nodes with spatial and temporal relationships, i.e., stronger edge. Light edges identify nodes with spatial relationships only.

\section{A case study: Information-Network for Illinois River}

A demonstrative example is presented in this section to illustrate the benefits of using the LTIN model to infer the emergent relationships at the 
outlet of the Illinois River, which is a single data node coming from the CI of GLTG. The area of the river basin and its tributaries encompasses 72,520 square kilometers, which is almost half the area of the state of Illinois. The river drains a large section of central Illinois and connects Lake Michigan with the Mississippi River (Figure 7). One of the primary tributaries of the Illinois River is the Sangamon River, which drains mostly rural agricultural areas in central Illinois. Agriculture is the predominant land use in the Illinois River Basin and it is the most important activity from the an economic and water-quality perspectives. The basin has highly productive and intensively cultivated farmland, which increases the application rates of nitrogen and phosphorus through fertilizers and manure to support the soybean and corn production. Runoff from these vast and flat farm areas carries a relatively high concentration of nutrients and pesticides into the small streams and major rivers of the basin. In addition, the contaminants from urban and industrial activities also impact the water quality of streams and rivers.

The legacy of agricultural drainage in tributaries of the Illinois River continues to influence the water quality and aquatic habitat in the Illinois River Basin. Different agricultural management practices have been developed to reduce nutrients from nonpoint sources (e.g., farms) to receiving tributaries. Within the basin of the Illinois River, multiple research groups and organizations are producing a collection of water quality parameters and streamflow monitoring information (Figure 7). Evaluating and understanding the effectiveness of agricultural management practices at a watershed scale requires the assemblage of water monitoring data, which are distributed across different CIs to trace the impact of each contaminant on the water quality in the 


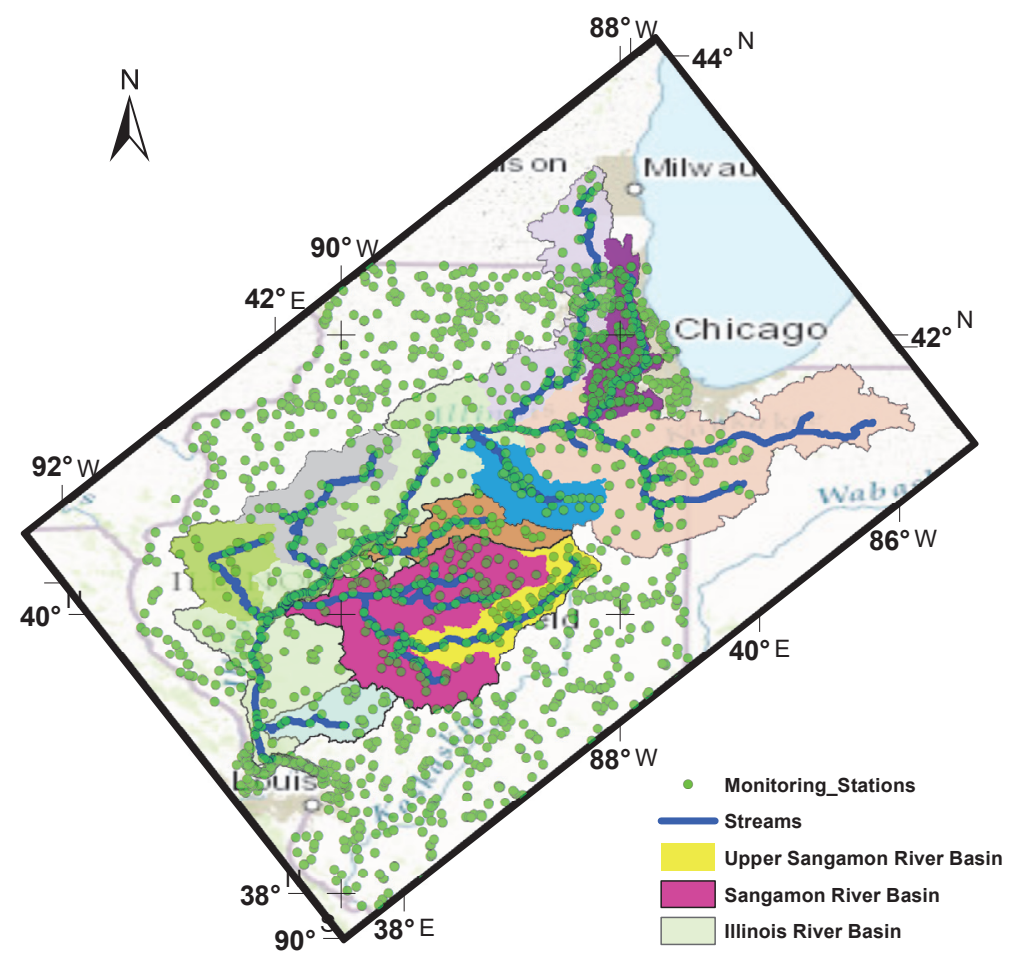

Figure 7: The spatial domain of the Illinois Basin and its tributaries, which include the Sangamon and Upper Sangamon watersheds. The circles represent the geographic distribution of 8900 water quality and streamflow monitoring stations in the state of Illinois. The information of these stations is distributed across many CIs.

Illinois River. Despite the availability of data nodes required to analyze the impact of agricultural practices on water quality parameters in the Illinois River basin, there is no straightforward approach to identify their CALs.

The information graph at the outlet of the Illinois River is extracted as a subgraph from the precomputed Information-Network (IN, http:// ecgs.ncsa.illinois.edu/gsis/read?graph=information_network). For demonstration purposes, we only represented two data nodes from the same CI, which are relevant for tracing the spatiotemporal variation of nitrate in the Illinois River. The rest of the edges and nodes are available in the 


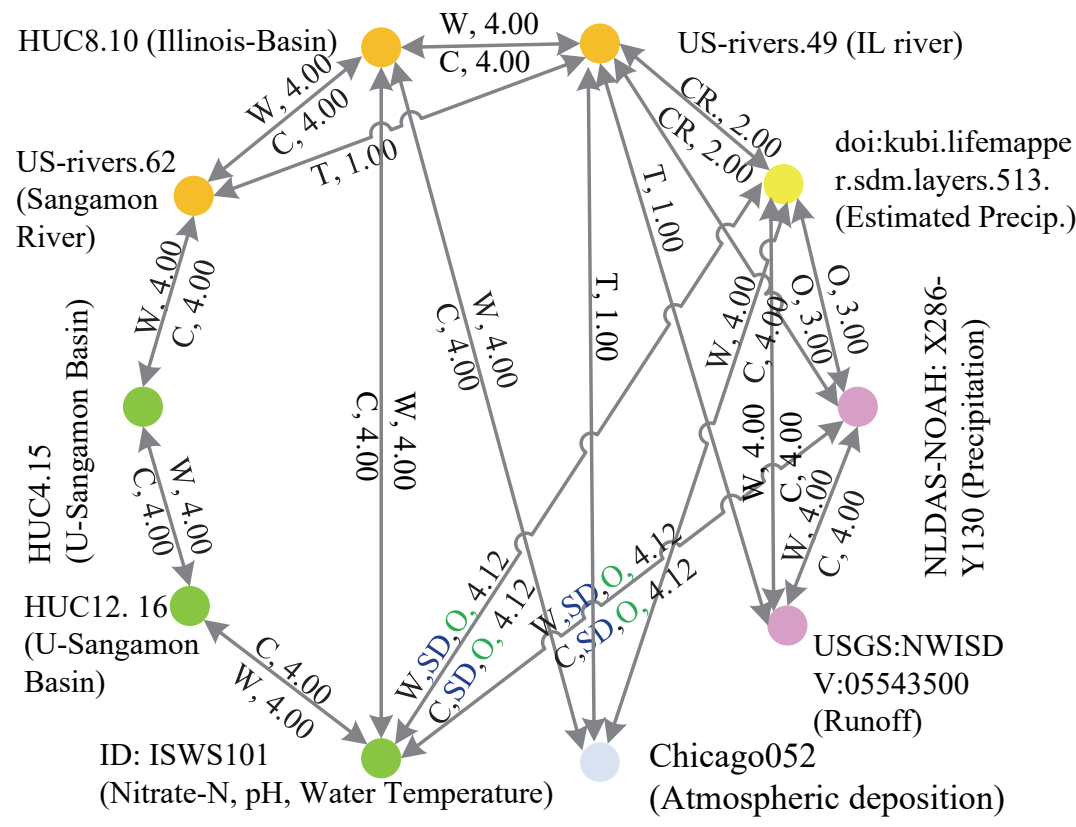

gp, TP, tp, Weight Edge

\begin{tabular}{|c|c|c|c|}
\hline $\begin{array}{l}\text { Source of Nodes } \\
\text { IML-CZO } \\
\text { SGGLM } \\
\text { CUASHI } \\
\text { GLTG } \\
\text { DataOne }\end{array}$ & $\begin{array}{l}\text { Geo-topological } \\
\text { Predicates (gp) } \\
\text { W: Within } \\
\text { C: Contains } \\
\text { T: Touches } \\
\text { CR: Crosses } \\
\text { O: Overlaps }\end{array}$ & $\begin{array}{l}\text { Temporal Pattern } \\
\text { (TP) } \\
\text { S: Sorting } \\
\text { ST: spatiotemporal } \\
\text { variation } \\
\text { SD: Spatiotemporal } \\
\text { dependency }\end{array}$ & $\begin{array}{l}\text { Temporal } \\
\text { Predicates (tp) } \\
\text { O: Overlaps } \\
\text { W:Withins } \\
\text { M: Meets } \\
\text { C: Contains }\end{array}$ \\
\hline
\end{tabular}

Figure 8: An excerpt of the Information-Network at the outlet of the Illinois River.

graph. Figure 8 demonstrates the role of the LTIN model in synthesizing of data nodes that have useful CALs, and do not have any prior connections with the Illinois River. For example, the information profile of the Illinois River (GLTG-GeoServer) is linked to the water quality observations ( IML-CZO: ISWS101) in the Sangamon Basin, which is an upstream measurement of water quality in the Illinois River. This link evolves through the spatial CAL between the Illinois River and the Sangamon River. Simi- 
larly, the edge between Illinois Basin and related climate monitoring station (SGGLM: Chicago 052) links the stream discharge to climate parameters. In addition, the Illinois River node is connected to a data node (DataOne: ORNL 173.3) that represents the climate parameters estimated on a monthly time scale: cloud cover, diurnal temperature range, precipitation, daily mean temperature, and vapor pressure. The connection between the Illinois River and runoff gauge (USGS: NWISD), which is coming from CUASHI-HIS, links streamflow-monitoring information with the information profile of the Illinois river. The information subgraph at the outlet of the Illinois River confirms the ability of the LTIN model to identify the CALs among nodes that may be useful in an analysis targeted at tracing the spatiotemporal variation of nitrate in the Illinois River while considering the daily atmospheric deposition, climate change, and historical streamflow data.

\section{Summary and Discussion}

Synthesizing data coming from diverse Earth science disciplines is necessary for improving long-tail data reusability and integration. In this study, we introduced the design of the Long-Tail Information-Network (LTIN) model, which provides a data synthesis solution based on the Linked Data approach. The model analyzes the information profiles associated with distributed data nodes to capture their Contextual Association Links (CALs), build a semantic Information-Network (IN), and annotate each data node with its CALs. LTIN model identifies the geo-topological and temporal-variable contextual association links among data nodes. The geo-topological context characterizes the spatial dependencies among data nodes. The temporal-variable 
context characterizes the temporal dependency pattern and the temporal topological relationships among data nodes with geo-topological relationships. The LTIN model uses a metadata-driven approach to build semantic information-networks among data nodes that are published over the web.

Similar to federated search engines, the LTIN model can search and retrieve information from multiple CIs, but with the additional advantage of inferred CALs among data nodes. The LTIN model automatically characterizes useful links among data nodes from their information profiles and curates these relationships in a semantic information graph. Using the LTIN model has some advantages in scientific knowledge discovery beyond that of typical federated search engines. These advantages include automatic aggregation of data nodes around environmental events, dynamic synthesis of data nodes, and provision of recommendations that can be approved or denied by domain expertise. In general, it can be said that the LTIN model transforms the knowledge discovery approach from depending on a scientist to define search queries to invoking automated sets of logic predicate rules that are triggered to identify dependencies and relationships among distributed data nodes. This approach minimizes the effort required from scientists to discover the dependencies and relationships among scientific events and unifies the methods of link prediction. The LTIN model is expandable and more logic rules can be added to analyze sophisticated metadata attributes through the engagement of larger scientific communities.

The LTIN model is designed to be easily extensible, and scalable with the number of CIs, web service requests, and storage of the resulting information graphs. Since the bottleneck in the scalability of the LTIN-Full version 
is the processing of information profiles through the binary link prediction layer, we have developed the precomputed information graph and thus the computation time is neglected during IN analysis. The graph is computed in a separate machine and uploaded to the graph database, where a RESTFul endpoint is provided for retrieving the network associated with specific data nodes. For LTIN-Lite, the primary scalability challenge is scaling up with the number of web service requests, which is typically resolved by making the services available on multiple machines, i.e., cloud environment. The LTIN-lite is hosted by Clowder [33, 36], which provides a Cloud infrastructure that is distributed across multiple physical and virtual machines and multiple execution environments. However, we acknowledge that the LTIN model may face scalability challenges as the number of the available data node increases. It will not be possible to conclude that the model is scalable until it has gone through additional testing from a wider set of users.

This work introduces the LTIN model and illustrates the potential benefits of building an information graph across distributed and heterogeneous CIs. While using the information graph has many advantages, there are also important barriers to consumption by third parties (e.g., models). Currently, users have to know the ID of the data node to retrieve its informationnetwork, and they must be aware of graph traversing approaches to manipulate the information graph. There are opportunities for abstracting the complexity of interfacing with the graph for end users, especially for automatic manipulation by third parties. Future research is needed to explore possibilities for more sophisticated graph traversing like those available in social networks, and track the provenance of changes of the edge generated by 
the LTIN model. Furthermore, models that are developed by small research groups and use long-tail data can parse the information-networks to identify and use data that are related to their models. In general, we see great potential in using the semantic information-networks coming out from the LTIN to improve the reusability of information across related scientific disciplines and to leverage the emergent information to the actionable knowledge level.

\section{Software availability}

Software name: Long Tail Information-Network (LTIN) model

Developer: Mostafa Elag/University of Illinois

Contact address: Ven Te Chow Hydrosystems Laboratory, Department of Civil and Environmental Engineering, University of Illinois, Urbana, IL, USA

E-mail: elag@illinois.edu

Year first available: 2015

Hardware required: Architecture independent

Required software: Windows/Linus

Program language: Python and JavaScript

Program size: $3 \mathrm{MB}$

Availability: Download under MIT License at:

LTIN model:https://github.com/mostafaelag/LTIN

Cost: Free 
670

\section{Acknowledgments}

Support from NSF grants "ACI-0940824", "ACI-1261582", "EAR1331906", and "ICER-1440315" are gratefully acknowledged.

[1] Adomavicius, G., Tuzhilin, A., 2011. Context-aware recommender systems. In: Recommender systems handbook. Springer, pp. 217-253.

[2] Al Hasan, M., Zaki, M. J., 2011. A survey of link prediction in social networks. In: Social network data analytics. Springer Berlin Heidelberg, pp. 243-275.

[3] Allard, S., 2012. Dataone: Facilitating escience through collaboration. Journal of eScience Librarianship 1 (1), 3.

680

[4] Allen, J. F., 1983. Maintaining knowledge about temporal intervals. Communications of the ACM 26 (11), 832-843.

[5] Allsopp, J., 2007. What are Microformats? Springer.

[6] Ames, D., Horsburgh, J., Goodall, J., Whiteaker, T., Tarboton, D., Maidment, D., 2009. Introducing the open source cuahsi hydrologic information system desktop application (his desktop). In: 18th World IMACS Congress and MODSIM09 International Congress on Modelling and Simulation, Modelling and Simulation Society of Australia and New Zealand and International Association for Mathematics and Computers in Simulation. pp. 4353-4359.

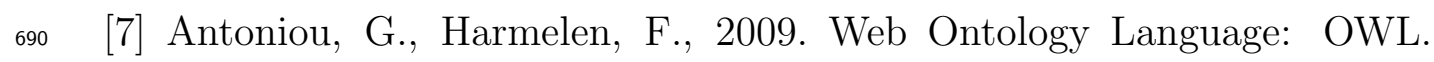
Handbook on ontologies, 91-110. 
[8] Bajcsy, P., 2008. A perspective on cyberinfrastructure for water research driven by informatics methodologies. Geography Compass 2 (6), 20402061.

[9] Brazier, P., Chebotko, A., Gonzalez, E., Kashlev, A., Piazza, A., 2010. Supporting geosciences web services metadata management and discovery. In: Services Computing (SCC), 2010 IEEE International Conference on. IEEE, pp. 625-626.

[10] Castronova, A. M., Goodall, J. L., Elag, M. M., 2013. Models as web services using the open geospatial consortium (ogc) web processing service (wps) standard. Environmental Modelling \& Software 41, 72-83.

[11] Clementini, E., Di Felice, P., 1995. A comparison of methods for representing topological relationships. Information Sciences-Applications 3 (3), 149-178.

[12] Elag M. M., Kumar P., Marini, L, and Peckham, S. D., Lui R, 2016. Semantic Interoperability of GeoScience long-tail resources over the Web, in Large-Scale Machine Learning in the Earth Sciences. In: Large-Scale Machine Learning in the Earth Sciences by A. N. Srivastava, R. Nemani, K. Steinhaeuser (Editors), Chapman \& Hall/CRC Press.

[13] Elag, M., Goodall, J. L., 2013. An ontology for component-based models of water resource systems. Water Resources Research 49 (8), 5077-5091.

[14] Elag, M. M., Goodall, J. L., Castronova, A. M., 2011. Feedback loops and temporal misalignment in component-based hydrologic mod- 
eling. Water Resources Research 47 (12). http://www.agu.org/pubs/ crossref/2011/2011WR010792.shtml

[15] Fayyad, U., Piatetsky-Shapiro, G., Smyth, P., 1996. The kdd process for extracting useful knowledge from volumes of data. Communications of the ACM 39 (11), 27-34.

[16] Fensel, D., Facca, F., Simperl, E., Toma, I., 2011. Semantic Web Services. Springer.

[17] Foster, I., Katz, D. S., Malik, T., Fox, P., 2012. Wagging the long tail of earth science: Why we need an earth science data web, and how to build it. http://semanticommunity.info/@api/deki/files/ 13867/=079_Foster .pdf

[18] Gahegan, M., Adams, B., 2014. Re-envisioning data description using peirce's pragmatics. In: Geographic information science. Springer, pp. $142-158$.

[19] Heidorn, P. B., 2008. Shedding light on the dark data in the long tail of science. Library Trends 57 (2), 280-299.

730 [20] Herring, J., 2011. Opengis implementation standard for geographic information-simple feature access-part 1: Common architecture. OGC Document.

[21] Hey, T., Trefethen, A. E., 2005. Cyberinfrastructure for e-science. Science 308 (5723), 817-821. 
[22] Horsburgh, J. S., Aufdenkampe, A. K., Mayorga, E., Lehnert, K. A., Hsu, L., Song, L., Jones, A. S., Damiano, S. G., Tarboton, D. G., Valentine, D., et al., 2016. Observations data model 2: A community information model for spatially discrete earth observations. Environmental Modelling \& Software 79, 55-74.

[23] Horsburgh, J. S., 2014. Toward integrated environmental modeling using research data infrastructures. In: Ames, D.P., Quinn, N.W.T., Rizzoli, A.E. (Eds.), "Proceedings of the 7th International Congress on Environmental Modelling and Software", San Diego, California, USA.

[24] Horsburgh, J. S., Tarboton, D. G., Maidment, D. R., Zaslavsky, I., 2011. Components of an environmental observatory information system. Computers \& Geosciences 37 (2), 207-218.

[25] Khare, R., Çelik, T., 2006. Microformats: a pragmatic path to the semantic web. In: Proceedings of the 15th international conference on World Wide Web. ACM, pp. 865-866.

750 [26] Kuhn, W., 2012. Core concepts of spatial information for transdisciplinary research. International Journal of Geographical Information Science 26 (12), 2267-2276.

[27] Kumar, P., 2006. Hydroinformatics : data integrative approaches in computation, analysis, and modeling. Taylor \& Francis, Boca Raton.

[28] Kumar, P., 2015. Hydrocomplexity: Addressing water security and emergent environmental risks. Water Resources Research 51 (7), 58275838. 
[29] Leinfelder, B., Tao, J., Costa, D., Jones, M. B., Servilla, M., O’Brien, M., Burt, C., 2010. A metadata-driven approach to loading and querying heterogeneous scientific data. Ecological Informatics 5 (1), 3-8.

[30] Liben-Nowell, D., Kleinberg, J., 2007. The link-prediction problem for social networks. Journal of the American society for information science and technology 58 (7), 1019-1031.

[31] Maidment, D. R., 2002. Arc Hydro: GIS for water resources. Vol. 1. ESRI, Inc.

[32] Michener, W., Vieglais, D., Vision, T., Kunze, J., Cruse, P., Janée, G., 2011. Dataone: Data observation network for earth-preserving data and enabling innovation in the biological and environmental sciences. D-Lib Magazine 17 (1), 3.

[33] Myers, J., Hedstrom, M., Akmon, D., Payette, S., Plale, B. A., Kouper, I., McCaulay, S., McDonald, R., Suriarachchi, I., Varadharaju, A., Kumar, P., Elag, M., Lee, J., Kooper, R., Marini, L., Aug 2015. Towards sustainable curation and preservation: The sead project's data services approach. In: e-Science (e-Science), 2015 IEEE 11th International Conference on. pp. 485-494.

[34] Newman, M. E., 2001. The structure of scientific collaboration networks. Proceedings of the National Academy of Sciences 98 (2), 404-409.

[35] Olfat, H., Kalantari, M., Rajabifard, A., Senot, H., Williamson, I. P., 2013. A gml-based approach to automate spatial metadata updating. 

250.

[36] Padhy, S., Jansen, G., Alameda, J., Black, E., Diesendruck, L., Dietze, M., Kumar, P., Kooper, R., Lee, J., Liu, R., et al., 2015. Brown dog: Leveraging everything towards autocuration. In: Big Data (Big Data), 2015 IEEE International Conference on. IEEE, pp. 493-500.

[37] Piasecki, M., Ames, D., Goodall, J., Hooper, R., Horsburgh, J., Maidment, D., Tarboton, D., Zaslavsky, I., 2010. Development of an information system for the hydrologic community. In: Proceedings of the Ninth International Conference on Hydroinformatics, Tianjin, China.

[38] Ruddell, B. L., Zaslavsky, I., Valentine, D., Beran, B., Piasecki, M., Fu, Q., Kumar, P., 2014. Sustainable long term scientific data publication: Lessons learned from a prototype observatory information system for the illinois river basin. Environmental Modelling \& Software 54, 73-87.

[39] Ruizhen, Z. Z. Z., 2009. Design of architecture for etl based on metadatadriven [j]. Computer Applications and Software 6, 022.

[40] Sea Grant, E., 2000. Fundamentals of a Sea Grant extension program. Sea Grant.

[41] Singh, G., Bharathi, S., Chervenak, A., Deelman, E., Kesselman, C., Manohar, M., Patil, S., Pearlman, L., 2003. A metadata catalog service for data intensive applications. In: Supercomputing, 2003 ACM/IEEE Conference. IEEE, pp. 33-33. 
[42] Swan, A., Brown, S., et al., 2008. To share or not to share: Publication and quality assurance of research data outputs: Main report. Research Information Network.

[43] Tarboton, D., Horsburgh, J., Maidment, D., Whiteaker, T., Zaslavsky, I., Piasecki, M., Goodall, J., Valentine, D., Whitenack, T., 2009. Development of a community hydrologic information system. In: 18th World IMACS Congress and MODSIM09 International Congress on Modelling and Simulation, Modelling and Simulation Society of Australia and New Zealand and International Association for Mathematics and Computers in Simulation. pp. 988-994.

[44] Wagener, T., Sivapalan, M., Troch, P., McGlynn, B., Harman, C., Gupta, H., Kumar, P., Rao, P., Basu, N., Wilson, J., 2010. The future of hydrology: An evolving science for a changing world. Water Resource Research 46 (5), 1-10.

[45] Wang, C., Satuluri, V., Parthasarathy, S., 2007. Local probabilistic models for link prediction. In: Data Mining, 2007. ICDM 2007. Seventh IEEE International Conference on. IEEE, pp. 322-331.

[46] Zaslavksy, I., Williams, M., Aufdenkampe, A., Lehnert, K., Mayorga, E., Horsburgh, J., 2011. Data infrastructure for the critical zone observatories (czodata): an earthcube design prototype. National Science Foundation EarthCube White Paper: Designs Category.

[47] Zaslavsky, I., Whitenack, T., Williams, M., Tarboton, D., Schreuders, K., Aufdenkampe, A., 2011. The initial design of data sharing infras- 
tructure for the critical zone observatory. In: Proceedings of the Environmental Information Management Conference, Santa Barbara, CA. pp. 28-29. 